University of Nebraska - Lincoln DigitalCommons@University of Nebraska - Lincoln

U.S. Air Force Research

U.S. Department of Defense

2013

\title{
Minimum-mass panels under probabilistic aeroelastic flutter constraints
}

Bret Stanford

Wright-Patterson AFB

Philip Beran

Wright-Patterson AFB

Follow this and additional works at: http://digitalcommons.unl.edu/usafresearch

Cart of the Chemical Engineering Commons

Stanford, Bret and Beran, Philip, "Minimum-mass panels under probabilistic aeroelastic flutter constraints" (2013). U.S. Air Force Research. 77.

http://digitalcommons.unl.edu/usafresearch/77

This Article is brought to you for free and open access by the U.S. Department of Defense at DigitalCommons@University of Nebraska - Lincoln. It has been accepted for inclusion in U.S. Air Force Research by an authorized administrator of DigitalCommons@University of Nebraska - Lincoln. 


\title{
Minimum-mass panels under probabilistic aeroelastic flutter constraints
}

\author{
Bret Stanford*, Philip Beran \\ U.S. Air Force Research Laboratory, Wright-Patterson AFB, OH 45433, United States
}

\section{A R T I C L E I N F O}

\section{Article history:}

Received 30 August 2012

Received in revised form

27 February 2013

Accepted 14 March 2013

Available online 8 April 2013

Keywords:

Aeroelastic flutter

Reliability-based design optimization

Elastic panels

\begin{abstract}
A B S T R A C T
Aeroelastic flutter is a dangerous failure mode, and aircraft structural components are designed under a deterministic flutter margin. Meeting this safety factor may result in overly-conservative structures, however, an alternative approach incorporates uncertainties into the computational models, and imposes a maximum allowable flutter probability during the optimization process. This technique is demonstrated for the variable-thickness design of an elastic panel subjected to supersonic flow. A performance measure approach based on the first-order reliability method incorporates probabilistic flutter constraints during the search for a minimum-mass panel. Optimization results are given for uncertainties in the panel's boundary conditions, and for non-deterministic thickness design variables.
\end{abstract}

Published by Elsevier B.V.

\section{Introduction}

The design and optimization of aerospace structures typically reflects a strong conflict between the continual desire to lower vehicle weight and cost, and requirements for a certain level of safety and reliability. Aeroelastic flutter, wherein a certain phasing of elastic, inertial, and aerodynamic loads causes a structure to oscillate in an unbounded manner (i.e., dynamically unstable), is of particular concern from a safety vantage point. The flutter speed occurs at a Hopf-bifurcation: the system damping characteristics are such that, at flight speeds even slightly above this point, the structural deformation may grow to unacceptably dangerous levels within a few vibration cycles [1]. Clearly, aerospace structures must be certified to operate in a manner that is free of flutter. The abrupt and explosive nature of flutter necessitates a safety or flutter margin (15\% for U.S. military aircraft [2]). During an optimization search for low-mass (and inevitably, low-stiffness) structures, it seems likely that satisfaction of this constraint margin will drive the design process.

Designing an aeroelastic structure under a pre-determined flutter margin is hardly the most efficient method for exploiting the tension between weight and safety, particularly in light of the inherently uncertain environment that these structures operate within. As reviewed by Danowsky et al. [3], geometry, inertial, material, and damping properties, flow conditions, surface roughness, and mechanical joint properties are all identified as critical

\footnotetext{
* Corresponding author. Tel.: +1937 2558286.

E-mail address: bretkennedystanford@gmail.com (B. Stanford).
}

sources of uncertainty. These uncertainties may be directly incorporated into the computational aeroelasticity model (provided they are parameter-based aleatory uncertainties), and the deterministic constraint upon the flutter margin $(15 \%$, e.g.) may be replaced by a constraint upon the probability that the structure will flutter: a probability of failure. The resulting reliability-based design optimization (RBDO) problem built upon risk-based constraints can help avoid the ultimate production of inefficient, overly-conservative vehicles.

The most popular methods in the literature for quantifying the probabilistic behavior of aeroelastic systems are sampling-based schemes. Traditional Monte Carlo-based simulation is typically too expensive on its own (though examples are given by Lindsley et al. [4] and Yi and Zhichun [5]), but it may be outfitted with various approximation methods in order to reduce the cost. These include response surface methods [6-8,3], stochastic projections [9-12], and reduced order modeling $[13,14,3]$. The focus of the current work is on aeroelastic structures with a very large number of design and/or uncertain parameters; analysis methods built upon approximation/expansion concepts typically become infeasible for high-dimensionality problems.

Perturbation methods are well-suited for these types of problems, however: the most popular is the first-order reliability method (FORM) [15]. General perturbation methods for quantification of aeroelastic uncertainty are utilized by Liaw and Yang [16] (mean-centered second moment method) and Castravete and Ibrahim [17] (Karhunen-Love expansions), while FORM is specifically used by Yang and Nikolaidis [18] and Verhoosel et al. [19]. FORM estimates the probability of failure $P_{f}$ by locating the most probable point (MPP) along a failure surface transformed into the 
independent standard normal random space. In this space, the distance between the origin and the MPP is the reliability index $\beta$. The failure probability is then approximated as $P_{f}=\Phi(-\beta)$ (where $\Phi$ is the cumulative distribution function (CDF) of the standard normal distribution), which is exactly true if the failure surface is linear and the random variables are jointly normal; errors are introduced otherwise [15].

FORM provides the probability that an aeroelastic design will fail (flutter, in this work), and the analytical sensitivity of $P_{f}$ can then be computed with respect to a large number of design variables, facilitating a gradient-based RBDO. This is demonstrated in the work of Yang and Nikolaidis [18], Pettit and Grandhi [20], Allen and Maute [21,22], Nikbay et al. [23], and Stanford and Beran [24], which address important aeroelastic issues such as gust response, aileron effectiveness, aerodynamic efficiency, elastic stresses, and limit cycle oscillations; none include flutter instabilities. In an optimization context, FORM may be implemented using either a reliability index approach (RIA) or a performance measure approach (PMA) [25], where the latter is known to be more robust and efficient, particularly when the random variables are not normally distributed [26].

The aeroelastic system considered here is well-known: flutter of a rectangular panel with supersonic flow over the top surface. Important early work in this subject is given by Dowell [27], and a more recent review of the panel literature is given by Mei et al. [28]. Specifically, it is desired to parameterize the thickness distribution throughout the plate, and minimize its mass under a probabilistic flutter constraint. For deterministic problems, this problem (or a close variant: maximize the flutter speed under a mass constraint) has been studied in the literature: Weisshaar [29], Peirson [30], Van Keuren and Eastep [31], Beiner and Librescu [32], and Barboni et al. [33]. An additional issue related to gradient-based flutter optimization (reliability-based or otherwise) is the potential discontinuity in the design space posed by flutter mode switching. The current work uses the frequencyseparation method advocated in Refs. [34,35], though aeroelastic damping-based methods have been utilized as well [36].

This work will assess the impact of a risk-based flutter constraint upon the thickness distribution and the minimum attainable mass. This is done for non-normally-distributed uncertain boundary conditions (separate random variables and deterministic design variables) and uncertain thickness distributions (non-deterministic design variables).

\section{Panel flutter}

The aeroelastic panel considered in this work is seen in Fig. 1. Both the length (chord) and the width of the square panel are $a$, and the uniform baseline thickness is $h_{o}=a / 190.5$. The panel is discretized into 3200 triangular finite elements, and as will be discussed below, the thickness of each element is allowed to increase or decrease by some percentage of $h_{o}$ during the optimization process. The plate is pinned (simply supported) along all four edges, and torsional springs are attached to each finite element node along the boundary, as seen in Fig. 1(b). If the spring stiffness is high, a clamped boundary condition is emulated. The upper surface of the panel is subjected to supersonic flow (flow density $\rho_{\infty}$, flow velocity $U_{\infty}$, and Mach number $M_{\infty}>1$ ) traveling along the $x$-axis.

\subsection{Equations of motion}

The transverse deflection $w$ of the (uniform thickness) plate is given as [27]:

$D \cdot \nabla^{4} w+\rho_{m} \cdot h_{0} \cdot \frac{\partial w}{\partial t}=\frac{\rho_{\infty} \cdot U_{\infty}^{2}}{\sqrt{M_{\infty}^{2}-1}} \cdot\left(\frac{\partial w}{\partial x}+\frac{M_{\infty}^{2}-2}{M_{\infty}^{2}-1} \cdot \frac{1}{U_{\infty}} \cdot \frac{\partial w}{\partial t}\right)$

where $\rho_{m}$ is the density of the plate, $t$ is time, and the bending stiffness is given by $D=E \cdot h_{o}^{3} /\left(12 \cdot\left(1-\nu^{2}\right)\right)$, where $E$ is the elastic modulus and $v$ is Poisson's ratio. Corrections for lower Mach numbers are given in the aerodynamic terms on the right side of Eq. (1); for $M_{\infty}$ much larger than unity, these terms coincide with the linear first-order piston theory originally given in Ref. [37].

The plate is then discretized into a series of planar triangular finite elements, as seen in Fig. 1. Three transverse degrees of freedom $\{w \quad \partial w / \partial x \quad \partial w / \partial y\}$ are assigned to each node (at each element vertex), which are then assembled into a global vector of unknowns, $\boldsymbol{u}$. In keeping with a simply-supported boundary condition, displacements $(w)$ along the edge are assumed to be zero, and are thus removed from $\boldsymbol{u}$. Eq. (1) can be converted into finite element form via the usual methods [38], and the result is

$\boldsymbol{M} \cdot \ddot{\boldsymbol{u}}+\boldsymbol{C} \cdot \dot{\boldsymbol{u}}+\left(\boldsymbol{K}+\boldsymbol{K}_{s}\right) \cdot \boldsymbol{u}=\boldsymbol{F}(\boldsymbol{u}, \dot{\boldsymbol{u}})$

$\boldsymbol{M}$ is a consistent mass matrix and $\boldsymbol{K}$ is a stiffness matrix (discrete Kirchhoff triangle [38] plate bending elements) obtained by assembling over each finite element $(e)$ :

$\boldsymbol{K}=\sum_{e}\left(\boldsymbol{K}_{e} \cdot h_{e}^{3}\right) \quad \boldsymbol{M}=\sum_{e}\left(\boldsymbol{M}_{e} \cdot h_{e}\right)$

where $h_{e}$ is the thickness of each finite element, and $\boldsymbol{K}_{e}$ and $\boldsymbol{M}_{e}$ are the elemental matrices associated with a unit-thickness plate. $\boldsymbol{K}_{s}$ is an additional stiffness matrix which reflects the contribution of the torsional springs along the plate boundary. This matrix is very sparse, with non-zero nodal stiffness only along the diagonal, corresponding to $\partial w / \partial x$ (leading and trailing edge) or $\partial w / \partial y$ (plate sides) degrees of freedom at nodes along the plate edges:

$\boldsymbol{K}_{s}=\sum_{n_{\mathrm{LE}}, n_{T E}}\left(k_{n} \cdot\left[\begin{array}{lll}0 & 0 & 0 \\ 0 & 1 & 0 \\ 0 & 0 & 0\end{array}\right]\right)+\sum_{n_{\text {sides }}}\left(k_{n} \cdot\left[\begin{array}{lll}0 & 0 & 0 \\ 0 & 0 & 0 \\ 0 & 0 & 1\end{array}\right]\right)$

where $k_{n}$ is the stiffness of each torsional spring, and $n_{\mathrm{LE}}, n_{\mathrm{TE}}$, and $n_{\text {sides }}$ are the finite element nodes along the leading edge, trailing
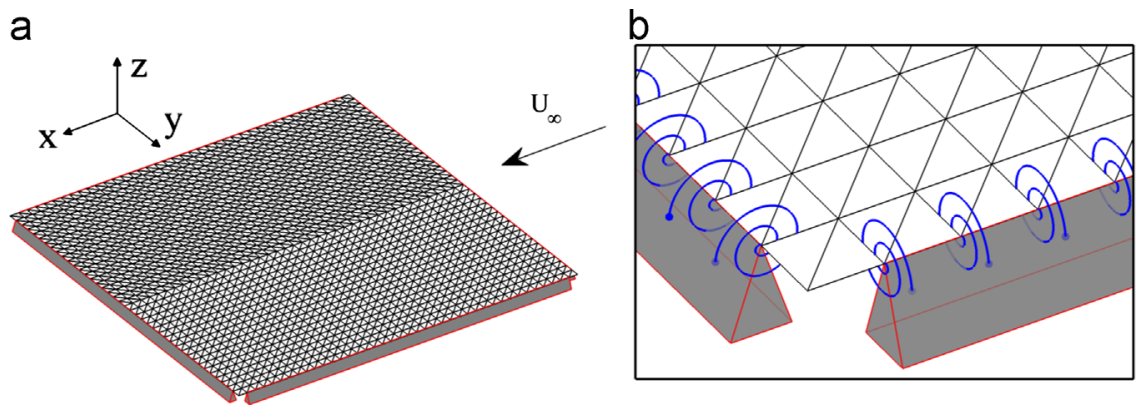

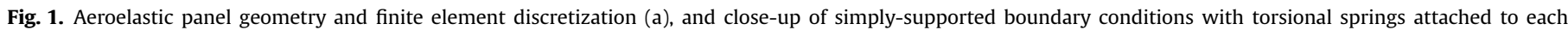
node (b). 
edge, and plate sides, respectively. The structural damping matrix is assumed to be proportional damping, given as $\boldsymbol{C}=\alpha_{c} \cdot \boldsymbol{M}+\beta_{c} \cdot\left(\boldsymbol{K}+\boldsymbol{K}_{s}\right)$, where $\alpha_{c}$ and $\beta_{c}$ are mass- and stiffness-proportional constants. It should be noted that neither the effect of $\boldsymbol{C}$ nor $\boldsymbol{K}_{s}$ are included in Eq. (1).

Via the right-hand-side of Eq. (1), the aerodynamic pressure is assumed to be spatially-constant within each finite element:

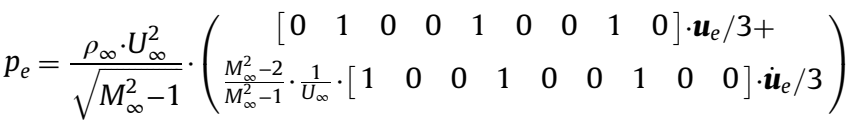

where $\boldsymbol{u}_{e}$ are the 9 degrees of freedom associated with each triangular element, and $\dot{\boldsymbol{u}}_{e}$ is the time derivative of that vector. It is noted that airloads over the panel only react to structural deformation $\left(\boldsymbol{u}_{e}\right)$ and deformational velocity $\left(\dot{\boldsymbol{u}}_{e}\right)$; additional aerodyanmics due to the thickness variations considered below are ignored. This is perhaps justified by assuming a thin flat foam layer is sealed to the top of the metallic panel, forming an outer mold line which ultimately communicates with the flow.

A work-equivalent aerodynamic force vector for each element is computed as

$\boldsymbol{F}_{e}=-p_{e} \cdot A_{e} \cdot\left[\begin{array}{lllllllll}1 & 0 & 0 & 1 & 0 & 0 & 1 & 0 & 0\end{array}\right]^{T} / 3$

where $A_{e}$ is the area of each element, and the negative sign is indicative of the fact that positive aerodynamic pressure on the top surface will push the structure downward. The global force vector may be computed by assembling over each finite element, written as a linear combination of $\boldsymbol{u}$ and $\boldsymbol{u}$ :

$\boldsymbol{F}(\boldsymbol{u}, \dot{\boldsymbol{u}})=\sum_{e}\left(\boldsymbol{F}_{e}\right)=\sqrt{\lambda} \cdot \boldsymbol{C}_{a} \cdot \dot{\boldsymbol{u}}+\lambda \cdot \boldsymbol{K}_{a} \cdot \boldsymbol{u}$

The aerodynamic stiffness and damping matrices are assembled as

$$
\begin{aligned}
& \boldsymbol{K}_{a}=\frac{-D}{9 \cdot a^{3}} \cdot \sum_{e}\left(A_{e} \cdot\left[\begin{array}{lllllllll}
1 & 0 & 0 & 1 & 0 & 0 & 1 & 0 & 0
\end{array}\right]^{T}\right. \\
& \left.\cdot\left[\begin{array}{lllllllll}
0 & 1 & 0 & 0 & 1 & 0 & 0 & 1 & 0
\end{array}\right]\right) \\
& \boldsymbol{C}_{a}=\frac{-\rho_{\infty} \cdot\left(M_{\infty}^{2}-2\right)}{9 \cdot\left(M_{\infty}^{2}-1\right)^{3 / 2}} \cdot\left(\frac{D \cdot \sqrt{M_{\infty}^{2}-1}}{\rho_{\infty} \cdot a^{3}}\right)^{1 / 2} \\
& \cdot \sum_{e}\left(A_{e} \cdot\left[\begin{array}{lllllllll}
1 & 0 & 0 & 1 & 0 & 0 & 1 & 0 & 0
\end{array}\right]^{T}\right. \\
& \left.\cdot\left[\begin{array}{lllllllll}
1 & 0 & 0 & 1 & 0 & 0 & 1 & 0 & 0
\end{array}\right]\right)
\end{aligned}
$$

The aerodynamic pressure parameter is defined as [27]

$\lambda=\frac{\rho_{\infty} \cdot U_{\infty}^{2} \cdot a^{3}}{D \cdot \sqrt{M_{\infty}^{2}-1}}$

The equations of motion, Eq. (2), of the aeroelastic panel can then be written as

$$
\boldsymbol{M} \cdot \ddot{\boldsymbol{u}}+\left(\boldsymbol{C}-\sqrt{\lambda} \cdot \boldsymbol{C}_{a}\right) \cdot \dot{\boldsymbol{u}}+\left(\boldsymbol{K}+\boldsymbol{K}_{s}-\lambda \cdot \boldsymbol{K}_{a}\right) \cdot \boldsymbol{u}=0
$$

A characteristic frequency of Eq. (11) (the first un-damped bending vibration frequency) is

$\omega_{o}=2 \cdot \pi^{2} \cdot \sqrt{D /\left(\rho_{m} \cdot h_{o} \cdot a^{4}\right)}$

and the mass ratio of the aeroelastic system is

$\mu=\rho_{\infty} \cdot a /\left(\rho_{m} \cdot h_{o}\right)$

Defining the vector $\boldsymbol{q}=\left\{\begin{array}{ll}\boldsymbol{u}^{T} & \dot{\boldsymbol{u}}^{T}\end{array}\right\}^{T}$, Eq. (11) may be placed in standard first-order form

$\left[\begin{array}{cc}\mathbf{I} & 0 \\ 0 & \boldsymbol{M}\end{array}\right] \cdot\left\{\begin{array}{l}\dot{\boldsymbol{u}} \\ \ddot{\boldsymbol{u}}\end{array}\right\}=\left[\begin{array}{cc}0 & \mathbf{I} \\ -\boldsymbol{K}-\boldsymbol{K}_{s}+\lambda \cdot \boldsymbol{K}_{a} & -\boldsymbol{C}+\sqrt{\lambda} \cdot \boldsymbol{C}_{a}\end{array}\right] \cdot\left\{\begin{array}{l}\boldsymbol{u} \\ \dot{\boldsymbol{u}}\end{array}\right\}$
$\boldsymbol{A} \cdot \dot{\boldsymbol{q}}=\boldsymbol{B} \cdot \boldsymbol{q}$

An eigenproblem based on Eq. (15) is written as

$\left(\boldsymbol{B}-\beta_{k} \cdot \boldsymbol{A}\right) \cdot \boldsymbol{\psi}_{k}^{R}=0$

where $\boldsymbol{\psi}_{k}^{R}$ is the right eigenvector associated with the $k$ th eigenvalue $\beta_{k}$, both of which, in general, will be complex.

For the results given below, $\mu / M_{\infty}=0.1$ and the damping parameters are set as $\alpha_{c}=10 \mathrm{~s}^{-1}$ and $\beta_{c}=10^{-4} \mathrm{~s}$. It should be noted that the parameters used to define the global behavior of the panel $\left(D, \lambda, \omega_{0}, \mu\right)$ are always computed with the baseline uniform thickness $h_{o}$, despite the fact that, during structural optimization, the thickness of each individual element $\left(h_{e}\right)$ is allowed to deviate from this value. Finally, the equations of motion as written in this section consider only half of the panel geometry in Fig. 1, with symmetric boundary conditions assumed along the centerline $(\partial w / \partial y=0)$. In a stochastic environment, random asymmetries in the panel structure are likely, thus weakening this assumption. For the reliability-based methods however, the MPP will only reflect an asymmetric structure if some variability is assumed in the flow vector (i.e., a random yaw angle), which has not been considered here.

\subsection{Direct flutter computations}

Flutter is defined as a loss of dynamic stability of the equations of motion (Eq. (15)) about an equilibrium solution. For this work, because the angle of attack of the flow vector is zero, and because the panel has no curvature (camber), the equilibrium solution is the trivial vector: $\boldsymbol{q}_{e}=0$. Flutter occurs at a Hopf-bifurcation point: for increasing values of $\lambda$ (since flow speed and Mach number are treated separately, this is an unmatched analysis), a pair of complex conjugate eigenvalues $\beta_{k}$ of the system cross the imaginary axis. Defining the aeroelastic damping of each mode as $g_{k}=\operatorname{Re}$ $\left(\beta_{k}\right)$, the system loses stability (perturbations grow exponentially with time) via flutter when $g_{k}$ of any mode becomes positive [1]. The damping of the composite response, $G$, corresponds to the eigenvalue with the largest real part (the most unstable mode): $G=\max \left(g_{k}\right)$. The flutter point is the point where $G=0$, and occurs at $\lambda=\lambda^{*}$; the imaginary portion of this critical eigenvalue at $\lambda^{*}$ is the flutter frequency, $\omega^{*}$.

This process is demonstrated in Fig. 2 for a panel with a uniform thickness $h_{o}$ and no torsional springs around the panel's perimeter (simply-supported), where it is understood that only the positive imaginary portions of each complex conjugate are shown. The eigenvalues at $\lambda=0$ (wind off) are the uncoupled vibration eigenvalues of the system, and $\operatorname{Re}\left(\beta_{k}\right)$ is entirely governed by the structural damping $\boldsymbol{C}$. The imaginary part of the first eigenvalue at this point is equal to $\omega_{o}$, and so the normalized quantity is unity. The damping of each mode becomes more stable as the flow is increased until $\lambda=320$, at which point the damping of the first mode becomes less stable. The flutter point $\lambda^{*}$ is located at 533.9 (which matches well with the value plotted by Dowell [27] for a simply supported panel), and is characterized by a coalescence of the imaginary portion of the first two modes.

Several methods can be used for locating the flutter point in a direct manner (i.e., as the outcome of a process): most require an initial guess for $\lambda^{*}$. This can be obtained by a $\lambda$-marching method: $\lambda$ is set to 0 and the eigenvalues $\beta_{k}$ (Eq. (16)) are computed. If $G=\max \left(\operatorname{Re}\left(\beta_{k}\right)\right)$ is less than $0, \lambda$ is augmented by some $\Delta \lambda$, and the process is repeated until $G$ becomes positive. Provided that $\Delta \lambda$ is not too large, the value of $\lambda$ at which this occurs should be a reasonable approximation to $\lambda^{*}$. The repetitive eigenvalue computations during the search for $\lambda^{*}$ can be very costly however, particularly if $\Delta \lambda$ is of a moderate size. In an optimization context, the process may be conducted just once for the initial baseline 

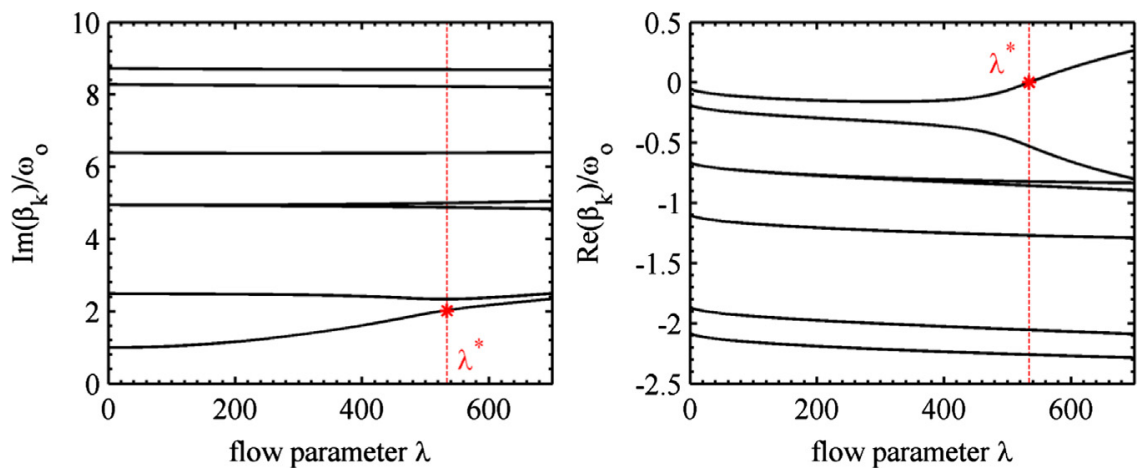

Fig. 2. Normalized eigenvalue migration for the uniform-thickness panel $\left(\boldsymbol{k}_{\boldsymbol{n}}=0\right)$.

design: a subsequent design iterate may use the previous design iterate's flutter point as an initial guess. It will be shown however, that eigenvalue information is needed in the range $0 \leq \lambda \leq \lambda^{*}$ to stabilize the flutter optimization problem, and so this $\lambda$-marching process is conducted for every design iterate.

Direct schemes for flutter point computations have been proposed in Refs. [39,40] using Hopf-point expansions, and in Ref. [41] using inverse power methods with shifting. A simpler method is utilized here by tracking the position of the least stable eigenmode, and uses Newton's recurrence formula to drive $G$ to zero:

$\lambda^{n+1}=\lambda^{n}-\bar{\omega} \cdot(\partial G / \partial \lambda)^{-1} \cdot G\left(\lambda^{n}\right)$

where $n$ is the iteration number. Solving the eigenproblem Eq. (16) at the current iterate $\lambda^{n}$ provides $G\left(\lambda^{n}\right)$, which is the damping of the least stable mode (if the $\lambda$-marching process detailed above is used, $G\left(\lambda^{0}\right)$ will be positive). The right and left eigenvectors may also be obtained $\left(\boldsymbol{\psi}_{k}^{R}, \boldsymbol{\psi}_{k}^{L}\right)$, and the derivative of the eigenvalues with respect to $\lambda$ is computed as [42]

$\frac{\partial \beta_{k}}{\partial \lambda}=\frac{\left(\boldsymbol{\psi}_{k}^{L}\right)^{T} \cdot\left(\frac{\partial \boldsymbol{B}}{\partial \lambda}-\beta_{k} \cdot \frac{\partial \boldsymbol{A}}{\partial \lambda}\right) \cdot\left(\boldsymbol{\psi}_{k}^{R}\right)}{\left(\boldsymbol{\psi}_{k}^{L}\right)^{T} \cdot \boldsymbol{A} \cdot\left(\boldsymbol{\psi}_{k}^{R}\right)}=\frac{\left(\boldsymbol{\psi}_{k}^{L}\right)^{T} \cdot \frac{\partial \boldsymbol{B}}{\partial \lambda} \cdot\left(\boldsymbol{\psi}_{k}^{R}\right)}{\left(\boldsymbol{\psi}_{k}^{L}\right)^{T} \cdot \boldsymbol{A} \cdot\left(\boldsymbol{\psi}_{k}^{R}\right)}$

where it can be seen in Eq. (14) that $\boldsymbol{A}$ has no dependence on $\lambda(\partial \boldsymbol{A} /$ $\partial \lambda=0)$. The damping slope $\partial G / \partial \lambda$ needed in Eq. (17) is computed as $\operatorname{Re}\left(\partial \beta_{k} / \partial \lambda\right)$, with the mode number $k$ corresponding to the least stable mode.

The region of convergence of Eq. (17) is wide above the flutter point $\left(\lambda^{n}>\lambda^{*}\right.$, where the unstable mode is clearly distinguished from the others, as seen in Fig. 2), but narrow below $\lambda^{*}$. As such, an under-relaxation factor $\bar{\omega}$ is typically needed to prevent overshoots that may drive the approximation well below the flutter point. Upon convergence of this system: $\lambda \rightarrow \lambda^{*}, \omega \rightarrow \omega^{*}, \beta_{k} \rightarrow \beta_{k}^{*}, \boldsymbol{\psi}_{k}^{R} \rightarrow \boldsymbol{\psi}_{k}^{R *}$, and $\boldsymbol{\psi}_{k}^{L} \rightarrow \boldsymbol{\psi}_{k}^{L *}$. It is unnecessary to compute eigenvalue information for mode numbers $k$ other then the least stable mode during the recurrence of Eq. (17). This information is needed for the optimization however, and can be computed by re-solving Eq. (16) at $\lambda^{*}$.

\subsection{Sensitivity analysis}

Relevant constraint-metrics for the RBDO studies given below are the flutter speed $\lambda^{*}$, as well as the eigenvalues $\beta_{k}$ for flow speeds in the range $0 \leq \lambda \leq \lambda^{*}$. A large number of random and design variables are considered here, necessitating analytical sensitivities of these metrics for both the reliability analysis (FORM) and the gradient-based optimization process. These are needed with respect to the thickness of each finite element $h_{e}$ and the stiffness of each torsional spring $k_{n}$. Derivations in this section are with respect to the thickness design variable vector $\boldsymbol{x}=\left\{\begin{array}{llll}h_{1} & h_{2} & \cdots & h_{E}\end{array}\right\}^{T}$. The formulas can also be used for the torsional spring derivatives, although it should be noted that the matrix $\boldsymbol{A}$ is a function of $h_{e}$ but not $k_{n}$, as the spring stiffness matrix $\boldsymbol{K}_{s}$ is only imbedded within $\boldsymbol{B}$.

If eigenvalue derivatives are desired at a given value of $\lambda$ (less than the flutter speed $\lambda^{*}$ ), these may be computed in a similar manner to Eq. (18):

$\frac{\mathrm{d} \beta_{k}}{\mathrm{~d} \boldsymbol{x}}=\frac{\left(\boldsymbol{\psi}_{k}^{L}\right)^{T} \cdot\left(\frac{\partial \boldsymbol{B}}{\partial \boldsymbol{x}}-\beta_{k} \cdot \frac{\partial \boldsymbol{A}}{\partial \boldsymbol{x}}\right) \cdot\left(\boldsymbol{\psi}_{k}^{R}\right)}{\left(\boldsymbol{\psi}_{k}^{L}\right)^{T} \cdot \boldsymbol{A} \cdot\left(\boldsymbol{\psi}_{k}^{R}\right)}$

where explicit derivatives of $\boldsymbol{A}$ and $\boldsymbol{B}$ are easily computed using Eqs. (3) and (4). If eigenvalue derivatives are desired at the flutter speed $\lambda^{*}$, then an implicit $\lambda$-dependence must be included in the chain rule:

$\frac{\mathrm{d} \beta_{k}^{*}}{\mathrm{~d} \boldsymbol{x}}=\frac{\left(\boldsymbol{\psi}_{k}^{L *}\right)^{T} \cdot\left(\frac{\partial \boldsymbol{B}^{*}}{\partial \boldsymbol{x}}-\beta_{k}^{*} \cdot \frac{\partial \boldsymbol{A}}{\partial \boldsymbol{x}}\right) \cdot\left(\boldsymbol{\psi}_{k}^{R *}\right)}{\left(\boldsymbol{\psi}_{k}^{L *}\right)^{T} \cdot \boldsymbol{A} \cdot\left(\boldsymbol{\psi}_{k}^{R *}\right)}+\frac{\partial \beta_{k}}{\partial \lambda} \cdot \frac{\partial \lambda^{*}}{\partial \boldsymbol{x}}$

where $\boldsymbol{B}^{*}$ is evaluated at the flutter point, and the derivative $\partial \beta_{k} / \partial \lambda$ is available from Eq. (18). The flutter point derivative $\partial \lambda^{*} / \partial \boldsymbol{x}$ is needed to complete Eq. (20), but also because $\lambda^{*}$ will be used as a constraint on its own. It may be computed by considering the aeroelastic damping at the flutter point, which is by definition zero regardless of the design/random variable vector:

$G^{*}=\max \left(\operatorname{Re}\left(\beta_{k}^{*}\right)\right)=0 \rightarrow \frac{\mathrm{d} G^{*}}{\mathrm{~d} \boldsymbol{x}}=\frac{\partial G^{*}}{\partial \boldsymbol{x}}+\frac{\partial G}{\partial \lambda} \cdot \frac{\partial \lambda^{*}}{\partial \boldsymbol{x}}=0$

$\frac{\partial \lambda^{*}}{\partial \boldsymbol{x}}=-\left(\frac{\partial G}{\partial \lambda}\right)^{-1} \cdot \frac{\partial G^{*}}{\partial \boldsymbol{x}}$

The term $\partial G / \partial \lambda$ is the real part of Eq. (18) (which has already been computed for use in Eq. (17) during the flutter point search), and the term $\partial G^{*} / \partial \boldsymbol{x}$ is the real part of Eq. (19), with the mode number $k$ in both equations corresponding to the flutter mode.

\section{Deterministic optimization}

Results for a series of deterministic flutter optimization cases are provided first, as a logical comparison to the RBDO cases discussed later. The optimization problem, solved with the method of moving asymptotes (MMA) [43] is stated as

$$
\begin{array}{ll}
\min & v . \\
\text { s.t. } & :\left\{\begin{array}{l}
\boldsymbol{x}_{\min }<\boldsymbol{x}<\boldsymbol{x}_{\max } \\
\lambda^{*} \geq \lambda_{c r}^{*} \\
\Delta \omega_{k} \geq \varepsilon_{\omega}
\end{array} \quad k=3, \ldots, N_{m}\right.
\end{array}
$$

The design variables for this problem are the thicknesses of each finite element: $\boldsymbol{x}=\left\{\begin{array}{llll}h_{1} & h_{2} & \cdots & h_{E}\end{array}\right\}^{T}$. Each thickness variable is 
constrained to lie between $\boldsymbol{x}_{\min }$ and $\boldsymbol{x}_{\max }$, which for this work are set to $0.5 \cdot h_{o}$ and $1.5 \cdot h_{o}$. The design variables are passed through a spatial filter prior to evaluation of Eq. (15) for eigenvalue and flutter computations. This is done via a linearly-decaying cone-shape function developed in Ref. [44] for topology optimization problems, with a radius equal to $6 \%$ of the panel length $a$. This restriction technique was found to provide smoothly-varying optimal thickness distributions, as intended.

The objective function in Eq. (23) is the volume of the panel $v$, which is normalized such that for a baseline uniform thickness panel $\left(h_{e}=h_{o}\right), v$ is equal to one. Since the material density is uniform, this effectively means that panel weight is being minimized. A constraint is placed on the minimum allowable flutter speed $\lambda_{c r}^{*}$. This threshold value is set equal to the flutter speed of the baseline design; no arbitrary flutter margin is imposed on the problem. Finally, there are a set of frequency separation constraints

$\Delta \omega_{k}=\min _{0 \leq \lambda \leq \lambda^{*}} \operatorname{Im}\left(\beta_{k}(\lambda)-\beta_{k-1}(\lambda)\right) \geq \varepsilon_{\omega}>0 \quad k=3, \ldots, N_{m}$

A well-known problem [34,35] in flutter-based structural optimization is mode-switching, where (during the optimization process) the critical mode switches from a frequency coalescence of modes 1 and 2 (as seen in Fig. 2) to a coalescence of modes 2 and 3, for example, or modes 3 and 4. This jump is a discontinuity in the design space and will prevent convergence of Eq. (23), particularly if two higher modes coalesce at a much lower $\lambda^{*}$ than that of the previous design iterate's [35]. Forcing the imaginary portions of the eigenvalues $\beta_{k}$ to be separated by some distance $\varepsilon_{\omega}$ at every value of $\lambda$ between 0 and the flutter speed will prevent mode switching, and the "nature" of the eigenvalue migration curves in Fig. 2 will remain unchanged [34]. A finite number of modes, $N_{m}$, are considered in this constraint.

The eigenvalues in Eq. (24) have been ordered based upon their imaginary parts: lowest to highest. The point of minimum separation between two modes $\left(\beta_{k-1}\right.$ and $\left.\beta_{k}\right)$ is $\lambda^{\Delta \omega_{k}}$, and may coincide with $\lambda^{*}$. If the $\lambda$-marching method described above is used to locate an initial guess for the flutter point, $\lambda^{\Delta \omega_{k}}$ and $\Delta \omega_{k}$ for each mode pair will be available at the end of this process. These will only be approximate values, but provided $\Delta \lambda$ is of a moderate size, their accuracy will be acceptable for the current purpose. The frequency separation $\Delta \omega_{2}$ is of no consequence (as interactions between modes 1 and 2 will always cause flutter, and $\lambda^{\Delta \omega_{2}}$ is always equal to $\lambda^{*}$ ), and so $k$ starts at 3 in Eq. (24). If, for two consecutive eigenvalues, this frequency separation is minimum at the flutter point $\left(\lambda^{\Delta \omega_{k}}=\lambda^{*}\right)$, then the constraint gradients may be computed with Eq. (20). If, on the other hand, the point of minimum separation occurs at any other point $\left(\lambda^{\Delta \omega_{k}}<\lambda^{*}\right)$, Eq. (19) is used. This is because Eq. (24) is a local minimum: $\partial\left(\Delta \omega_{k}\right) / \partial \lambda=0$, and so the second term in the chain rule of Eq. (20) is zero.

A series of optimal thickness distributions are given in Fig. 3, each corresponding to a different value of the torsional springs around the perimeter of the panel $k_{n}$ (normalized by the bending stiffness $D$ ). For these cases, the spring stiffness is spatially uniform around the plate, considered to be a known deterministic parameter, and is held fixed during the optimization. The majority of the thickness variables in Fig. 3 are at the lower $\left(0.5 \cdot h_{o}\right)$ or upper $\left(1.5 \cdot h_{o}\right)$ bounds, with a smooth topological transition between the two extremes, as ensured by the spatial filter [44]. The initial design for each of these optimization cases is the uniform thickness plate.

The results of the simply-supported case $\left(k_{n} / D=0\right)$ show two lobes of high thickness along the centerline ( $x$-axis) of the plate, which is qualitatively very similar to two-dimensional panel results published in Refs. [29,33]. Spanwise members attached to the sides of the plate are present as well, though these are removed in the optimal design for a slightly higher spring stiffness of $k_{n} / D=0.2$. Further increases in the torsional stiffness leads to a constant shift in the thickness distribution, with four lobes of high-thickness along the centerline for $k_{n} / D=4.0$ (which is high enough for the plate to be considered clamped along all sides). The topology at $k_{n} / D=0.4$ appears to be an outlier, however, with the centerline towards the trailing edge at the minimum thickness gage: the rearward lobe of high thickness has split into two. It is further noted that each structure in Fig. 3 shows asymmetries between the leading half and the trailing half of the panel, presumably due to the biasing effect of the flow vector.

Convergence histories for these six cases are given in Fig. 4, which plots the flutter speed $\lambda^{*}$ of the panel as a function of the objective $v$. For increasing values of torsional spring stiffness, the baseline designs are noted; each starts with a volume of unity (uniform thickness plate), while the flutter speed increases from $533.9\left(k_{n} / D=0\right)$ to $854.2\left(k_{n} / D=4.0\right)$. This latter value compares well with the flutter speed of a clamped panel drawn in Ref. [4]. The flutter speed of the baseline design is designated as the critical constraint boundary $\lambda_{c r}^{*}$. A dashed line is drawn at this value for each case in Fig. 4, below which the convergence history should not cross. For some of the cases $\left(k_{n} / D=0,0.2,1.2\right)$, the optimizer increases the volume during the first few iterations, which is due

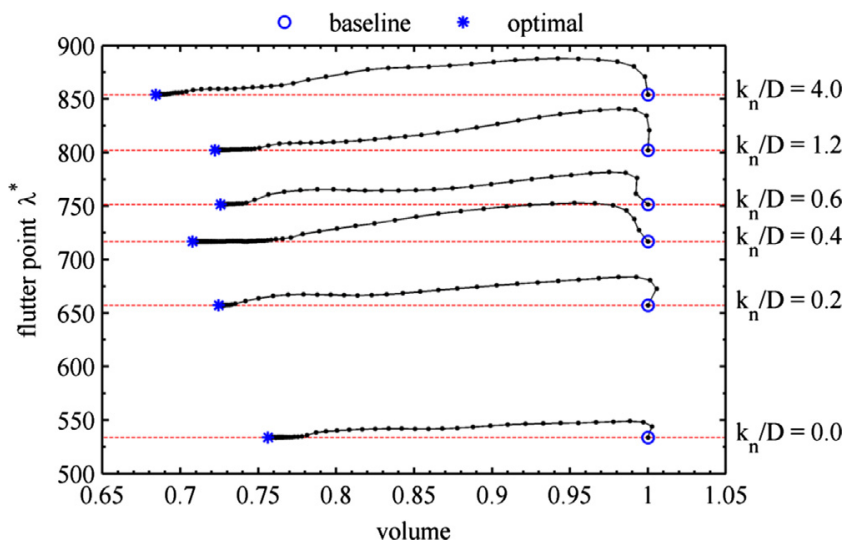

Fig. 4. Convergence histories for deterministic optimization.

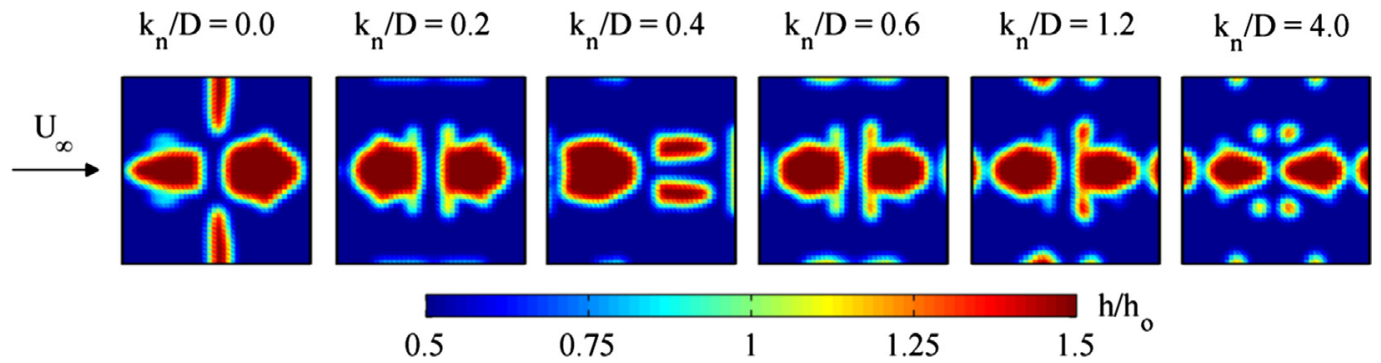

Fig. 3. Optimal (deterministic) thickness distributions for increasing stiffness of the torsional edge springs. 
to an initial violation in the frequency separation constraints (not shown). For the other cases, the optimizer is able to immediately decrease the volume and satisfy the frequency separation constraint simultaneously.

Beyond the first few iterations, convergence histories in Fig. 4 proceed from right to left, and the process is terminated when the change in the volume between two successive iterations is less $10^{-5}$. As would be expected, the flutter constraint is ultimately active for each case, such that the optimal design and the baseline design flutter at the same value of $\lambda$. The simply supported case $\left(k_{n} / D=0\right)$ is able to decrease the volume of the panel from 1 to 0.756 , whereas the clamped case $\left(k_{n} / D=4.0\right)$ decreases the volume to 0.684 . As above, the case of $k_{n} / D=0.4$ is fundamentally different from its adjacent cases: it has a lower volume, and takes far more iterations to converge than any other case (202, whereas the next highest is 167 iterations for $k_{n} / D=0$ ).

The convergence of the frequency separation constraints has not been shown, but these are typically active as well. Eigenvalues for both the baseline and the optimal panel structures are shown in Fig. 5 for $k_{n} / D=0$, where the uniform-thickness baseline data is repeated from Fig. 2. The two panels flutter at the same point (533.9), though the flutter frequency $\omega^{*}$ of the optimal panel is higher (despite lower natural vibration frequencies). The required frequency separation between two modes has been (arbitrarily) set to $\varepsilon_{\omega}=0.4 \cdot \omega_{o}$ for all of the cases in this paper. For the optimal design in Fig. 5, this constraint is clearly not active between modes 2 and 3 , but $\Delta \omega_{4}$ is active at $\lambda^{\Delta \omega_{4}}=260$ (well below the flutter speed), and $\Delta \omega_{5}$ is active at $\lambda^{\Delta \omega_{5}}=533.9$ (equal to the flutter speed).

The implication of these two constraints is that, if $\Delta \omega_{5}$ was dropped from the set of constraints, the critical flutter mode would begin to switch from a mode 1-2 coupling to a mode 4-5 coupling during the optimization process. This discontinuity would hinder further convergence, but both flutter mechanisms occur at about the same point. On the other hand, if $\Delta \omega_{4}$ were dropped, the flutter mode would switch to a mode 3-4 coupling, at which point the flutter speed would experience a drastic decrease to an infeasible flutter point $(\sim 260)$. This is demonstrated in Ref. [35]; the authors of that paper also note that lower values of $\varepsilon_{\omega}$ (i.e., making the constraint easier to satisfy) improves the performance of the optimal design, but decreases its robustness in terms of mode-switching and higher-mode flutter. These concepts will not be explored here, however.

\section{RBDO: uncertain boundary conditions}

As noted by Pettit [2], epistemic model-form uncertainties concerning the boundary conditions of a panel (idealized as simply supported) may be "converted" into a parameter-based aleatory uncertainty with the inclusion of the rotational springs along the panel boundary seen in Fig. 1 . Such a conversion comes with several caveats, of course, the most important of which is that details of the physical connection between the panel and its supports are lost, along with the exact stress distribution along the edges of the panel [2].

The random torsional stiffness of each spring $k_{n}$ is assumed to have a Weibull distribution, a choice made in Ref. [4] also. This distribution is entirely defined by a scale factor $\alpha_{k}$ and a shape factor $\gamma_{k}$ : a range of values for the scale factor are considered here, but the shape factor is fixed at unity. As such, the mean of the random variables is always equal to $\alpha_{k}$, the probability density function (PDF) is maximum at $k_{n}=0$ (negative values of $k_{n}$ are obviously infeasible) and monotonically drops to zero as $k_{n}$ tends to infinity. Qualitatively, the panel is intended to be simplysupported, but (much) stronger boundary conditions are possible.

Before developing an RBDO framework, relationships between the Weibull distribution and panel flutter are explored. The random variable vector $\boldsymbol{r}$ is populated with two independent members: the torsional stiffness of every rotational spring along the leading and trailing edge of the panel $\left(r_{1}\right)$ and the stiffness of every spring along the panel sides $\left(r_{2}\right)$. Each is varied from a normalized value of $k_{n} / D=0$ to 2 , and the resulting flutter speed is plotted in Fig. 6(a). As above, $\lambda^{*}$ varies between 533.9 (simplysupported) and 854.2 (clamped). Then, assuming a scale factor $\alpha_{k} / D=0.8$ (which will also be the mean value, marked with a "+" in the figure), the vector $r$ is converted into standard normal space $\boldsymbol{u}[45]$ :

$\boldsymbol{u}=T(\boldsymbol{r}) \rightarrow u_{i}=\Phi^{-1}\left(1-\mathrm{e}^{-\left(r_{i} / \alpha_{k}\right)^{\gamma_{k}}}\right)$

Flutter results in $\boldsymbol{u}$-space are given in Fig. 6(b), where it is understood that a $u_{i}$ of $-\infty$ maps to a $r_{i}$ of 0 , and $u_{i}$ of $\infty$ maps to a $r_{i}$ of $\infty$, and the transformation $T$ is strongly nonlinear. A dashed line indicating a reliability index $\beta=\sqrt{\left(\boldsymbol{u}^{T} \cdot \boldsymbol{u}\right)}$ is drawn in the figure for an arbitrary value of 1 , and this circle can be mapped back into the physical space in Fig. 6(a). The point with the minimum flutter speed on the circle is the MPP, and is marked with an asterisk. The curvatures in the $\boldsymbol{u}$-space are moderate at this point, and so FORM should be fairly accurate in computing a failure probability [15]. The torsional stiffness along the sides of the panel $\left(r_{2}\right)$ has a weak effect upon the flutter speed however, and so the exercise is repeated by setting the stiffness along the sides equal to zero, letting $r_{1}$ be the leading edge stiffness, and $r_{2}$ be the trailing edge stiffness. The curvature at the MPP in this case (Fig. 7) is significant, and the computation $P_{f}=\Phi(-\beta)$ will underpredict the failure probability.

Despite these potential accuracy issues, FORM is commonly considered the best choice for complex physical problems with a
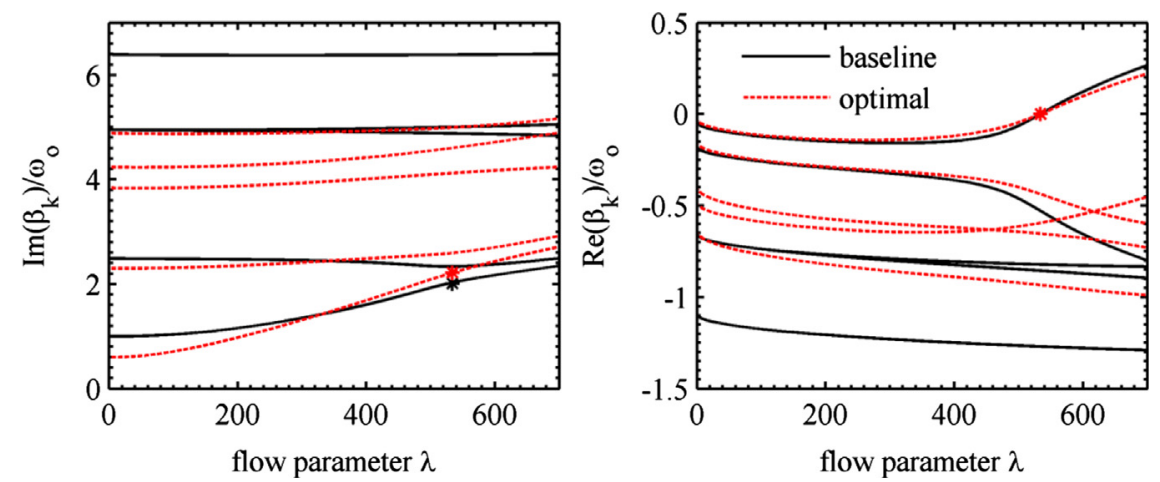

Fig. 5. Normalized eigenvalue migration for the uniform-thickness panel and the optimal panel $\left(k_{n}=0\right)$. 

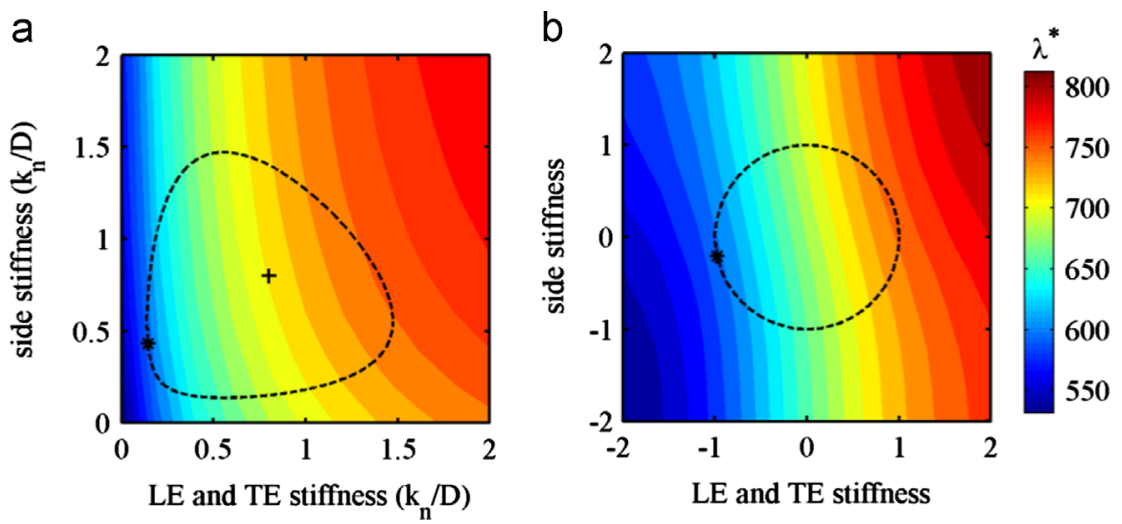

Fig. 6. Flutter speeds as a function of the leading/trailing edge and the panel side torsional spring stiffness: physical space (a) and standard normal space (b).
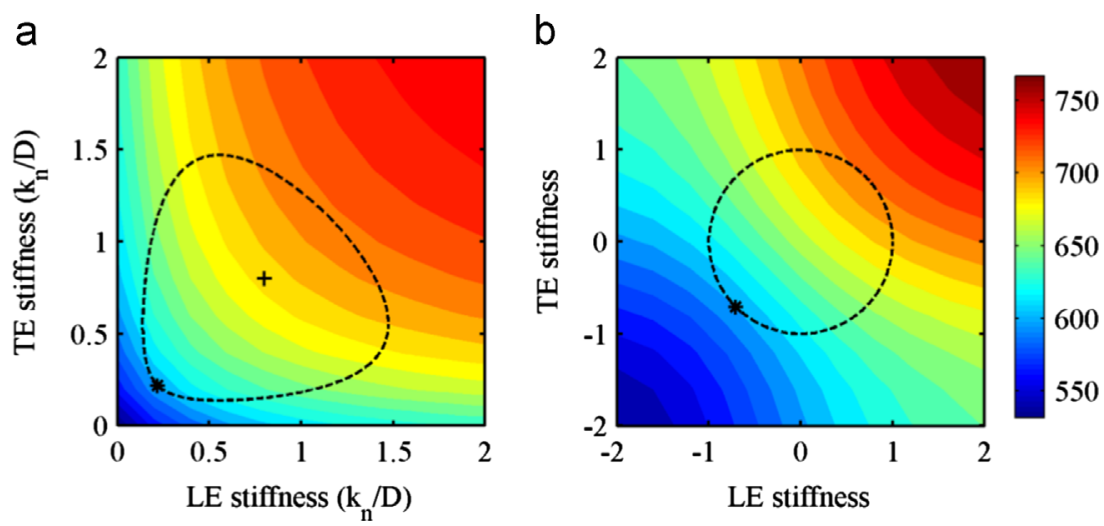

Fig. 7. Flutter speeds as a function of the leading and the trailing edge torsional spring stiffness: physical space (a) and standard normal space (b)

large number of variables [21], and will be used for all of the RBDO results presented here. Curvatures of the eigenvalue and flutter metrics could conceivably be computed for a second-order reliability analysis (SORM), as demonstrated by Verhoosel et al. [19], or alternatively could be approximated with quasi-Newton methods. For PMA-based SORM, however, the design-dependence between $\beta$ and $P_{f}$ induces a significant nonlinearity, and third-order gradients are needed for exact RBDO design derivatives [46]. Furthermore, all of the commonly-used SORM approximations have accuracy issues in various situations: negative curvature, saddle points, low reliability indices, large numbers of random variables, etc. [47], all of which the optimizer may exploit. Further studies are needed to assess whether the use of second-order RBDO methods would arrive at flutter-optimal designs significantly different than those obtained here.

Turning now to the development of the RBDO framework, this section considers a set of deterministic design variables $\boldsymbol{x}=\left\{\begin{array}{llll}h_{1} & h_{2} & \cdots & h_{E}\end{array}\right\}^{T}$, the thickness of each finite element, and an entirely separate set of random variables $\boldsymbol{r}=\left\{\begin{array}{llll}k_{1} & k_{2} & \cdots & k_{N}\end{array}\right\}^{T}$, which is the torsional stiffness of the rotational spring at each boundary node. These random variables are uncorrelated and independent, though a random field approximation (used for spatial variability in elastic modulus in Refs. $[4,17])$ may be more appropriate. For this work, spring stiffness variables are sent through the same spatial filter [44] used for thickness variables, prior to flutter computations, providing some level of realistic spatial correlation. This method precludes the need for more complex transformations [15] than used in Eq. (25), as the variables explicitly handled by FORM remain uncorrelated.

A performance function is $g(\boldsymbol{r}, \boldsymbol{x})$, where a negative value implies an unacceptable design (failure). For this work, failure occurs when the flutter speed becomes less than the desired threshold $\left(g=\lambda^{*}-\lambda_{c r}^{*}\right)$. Each design in Fig. 4 is "safe" in a deterministic sense, as the flutter constraint is always active, but the failure probability $P_{f}$ of each will be close to 0.5 . In order to improve their reliability, the PMA approach first identifies a target reliability index $\beta_{t}$. The following optimization problem is then solved in the $\boldsymbol{u}$-space [25] (with the design variables $\boldsymbol{x}$ held fixed):

$$
\min _{\boldsymbol{u}} g(\boldsymbol{u}, \boldsymbol{x}) \quad \text { s.t. }:\left\{\sqrt{\left(\boldsymbol{u}^{T} \cdot \boldsymbol{u}\right)}=\beta_{t}\right.
$$

This problem is efficiently solved with the conjugate mean-value method (CMV) [27], which is found to be superior to the generalpurpose MMA optimizer for these cases (as it is specifically tailored to solve Eq. (26)), and requires the gradient:

$\frac{\partial g}{\partial \boldsymbol{u}}=\frac{\partial g}{\partial \boldsymbol{r}} \cdot \frac{\partial \boldsymbol{r}}{\partial \boldsymbol{u}}$

The gradient $\partial g / \partial \boldsymbol{r}$ may be computed using the flutter derivative computation of Eq. (22) (replacing $\boldsymbol{r}$ for $\boldsymbol{x}$ in that equation), while $\partial \boldsymbol{r} / \partial \boldsymbol{u}$ reflects the inverse of the transformation given in Eq. (25): $\boldsymbol{r}=T^{-1}(\boldsymbol{u})$. The optimal point of Eq. (26) is $\boldsymbol{u}_{\beta=\beta_{t}}^{*}$ (the MPP) and the probabilistic performance measure is $g^{*}=g\left(\boldsymbol{u}_{\beta=\beta_{t}}^{*}, \boldsymbol{x}\right)$. An example of this process is demonstrated in Figs. 6 and 7, for problems with two random variables.

The RBDO problem is then formulated as

$$
\min _{\boldsymbol{x}} v \text { s.t. }:\left\{\begin{array}{l}
\boldsymbol{x}_{\min }<\boldsymbol{x}<\boldsymbol{x}_{\max } \\
g^{*} \geq 0 \\
\Delta \omega_{k} \geq \varepsilon_{\omega} \quad k=3, \ldots, N_{m}
\end{array}\right.
$$

This is identical to the deterministic Eq. (23), except that the flutter constraint has been replaced with a constraint that the performance measure remains positive. Like the flutter constraint, this constraint is expected to be active at the optimal design. The 
design gradients are computed as

$\frac{\mathrm{d} g^{*}}{\mathrm{~d} \boldsymbol{x}}=\frac{\partial g^{*}}{\partial \boldsymbol{x}}+\frac{\partial g^{*}}{\partial \boldsymbol{u}} \cdot \frac{\partial \boldsymbol{u}_{\beta=\beta_{t}}^{*}}{\partial \boldsymbol{x}}$

The first term in Eq. (29) is an explicit dependence; the second term is an implicit dependence via changes in the location of the MPP. It can be shown [22] via the optimality conditions of Eq. (26) that since the MPP is constrained to have a certain reliability index $\beta_{t}$, the performance measure does not depend on $\boldsymbol{u}$. As such, the total derivative can just be evaluated at the MPP: $\mathrm{dg}^{*} / \mathrm{d} \boldsymbol{x}=\partial \mathrm{g}^{*} / \partial \boldsymbol{x}$, which is computed using Eq. (22). This optimization problem, like the deterministic case, is solved using MMA.

Contrastingly, the RIA approach locates the closest point on the failure surface $(g=0)$, which is the MPP, and then optimizes such that this distance never becomes less than $\beta_{t}$. For very simple design spaces and random spaces, the PMA and the RIA approach will give the same optimal design $\boldsymbol{x}$ and the same MPP (Allen and Maute [22] show that the two approaches converge to essentially the same point for complex aeroelastic problems as well, though this should be problem dependent). The reliability problem in Eq. (26) is far less susceptible to the strong nonlinearities induced by the non-uniform random variables considered here [26], however.

It is also noted that the frequency separation constraints in Eq. (28) are still deterministic, constrained to be separated by an arbitrary margin $\varepsilon_{\omega}$. These constraints are largely numerical tools to ensure a smooth optimization process [34] rather than physical considerations, though larger values of $\varepsilon_{\omega}$ can be thought of as increasing the probability that the fundamental flutter mechanism remains an interaction between the first two modes (and as such, decreasing the probability that stochastic variations in the system parameters may lead to a drastic drop in the flutter speed). Furthermore, the value of $\varepsilon_{\omega}$ used here may be overly conservative, and so future work can consider probabilistic formulations of these constraints.

RBDO results for 4 cases are given in Fig. 8: scale factors $\alpha_{k} / D$ of $0.2,0.4,0.6$, and 1.2 . Two of the cases corresponding to those given in Fig. 3 could not be reproduced here in an RBDO context. For a scale factor of 0 , the transformation of Eq. (25) is not defined. For a value of 4.0, the CMV method was unable to solve Eq. (26), presumably due to the strong nonlinearities within the failure surface around $\boldsymbol{u}_{\beta=\beta_{t}}^{*}$. Focusing first on the RBDO result for $\alpha_{k} / D=0.2$, the deterministic optimum of Fig. 3 is used as an initial baseline design, and the flutter speed of this result (657.3) is set as the critical threshold $\lambda_{c r}^{*}$. This value, of course, is the same constraint boundary used during the deterministic optimization, as it is the flutter speed of the uniform-thickness panel. At the first iteration, the flutter speed of the design whose random variables are all set to their mean values $\left(k_{n}=\alpha_{k}\right)$ is 657.3 , but the flutter speed at the MPP $\left(\beta_{t}=3\right)$ is much lower: $\lambda_{\beta=\beta_{t}}^{*}=564.9$. As such, the probabilistic performance measure $g^{*}$ is -92.4 , and so the baseline design is infeasible, as expected.

Within four iterations, the optimizer is able to push the flutter speed of the MPP onto the $\lambda_{c r}^{*}$ constraint boundary $\left(g^{*}=0\right)$, and then spends another 50 iterations traveling along this boundary in an effort to decrease the panel volume. Upon convergence, the inclusion of uncertainty in the boundary conditions has increased this objective function by $3.94 \%$ over the deterministic design. The performance of the mean design (which is not provided to the optimizer at any point, but is shown here for completeness) has been increased above the constraint boundary to $\lambda^{*}=729.2$. The flutter margin for this case is $9.9 \%$, which is far lower than the (in this instance) overly-conservative value of $15 \%$ cited in Ref. [2]. Of course, if larger values of $\beta_{t}$ were required (smaller probability of failure), or Weibull distributions with a larger variability, this may no longer be the case.

The remaining three cases in Fig. $8\left(\alpha_{k} / D=0.4,0.6,1.2\right)$ proceed in a similar manner, where it is understood that these cases each use a different deterministic optimum (Fig. 3) as their baseline starting point. Setting the normalized scale factor to 0.4 forces the largest compromise between reliability and mass (5.31\% increase in mass), while the optimal design under $\alpha_{k} / D=1.2$ has the lowest flutter margin, where $\lambda^{*}$ of the mean design is only $6.3 \%$ above the critical value. Interestingly, each case spends the majority of the design iterations with the MPP directly at $\lambda_{c r}^{*}$, but during this time the flutter speed of the mean design decreases (substantially so for the higher scale factors). This is not explicitly done, as the meanflutter metric is never provided to the optimizer, but does indicate a fundamentally different set of design requirements between the two structures.

These differences are seen in Fig. 9, which gives the deterministic optimal thickness distribution (repeated from Fig. 3) on one side of each panel, the new reliability-based optimum on the other side, the distribution of torsional spring stiffness along the perimeter for the MPP of each design, and an indication of the mean spring stiffness (scale factor), for each of the four cases in Fig. 8. For a normalized shape factor of $\alpha_{k} / D=0.2$, topological differences between the deterministic structure and the reliabilitybased structure are minor, despite the fact that the latter has a much higher (deterministic) flutter speed. The reliability-based design has slightly increased the size of the two high-thickness lobes along the centerline, which accounts for the $3.94 \%$ increase in mass cited above. This increases the stiffness of the panel, and gives it a higher flutter reliability, as desired.

Slight increases in the topological features are evident in the RBDO results for the remaining three cases in Fig. 9 as well. Each of these deterministic structures allocates material directly at the centerline of the leading and trailing edges, where the thickness of
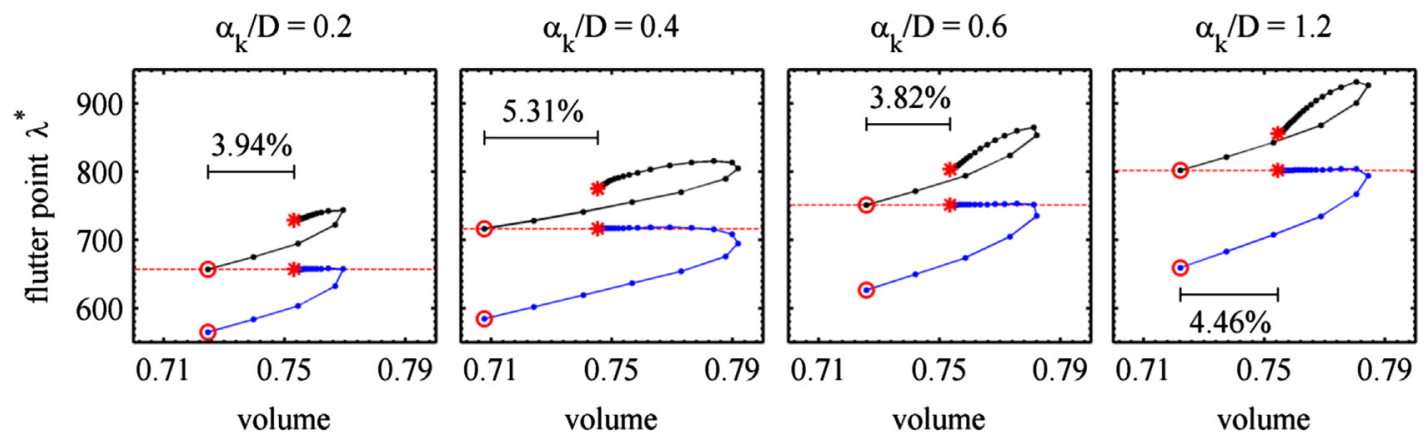

$\lambda_{\mathrm{cr}}^{*}$

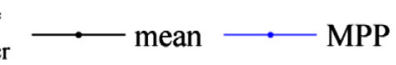

- baseline

* optimal

Fig. 8. Convergence histories for reliability-based design optimization with uncertain boundary conditions. 

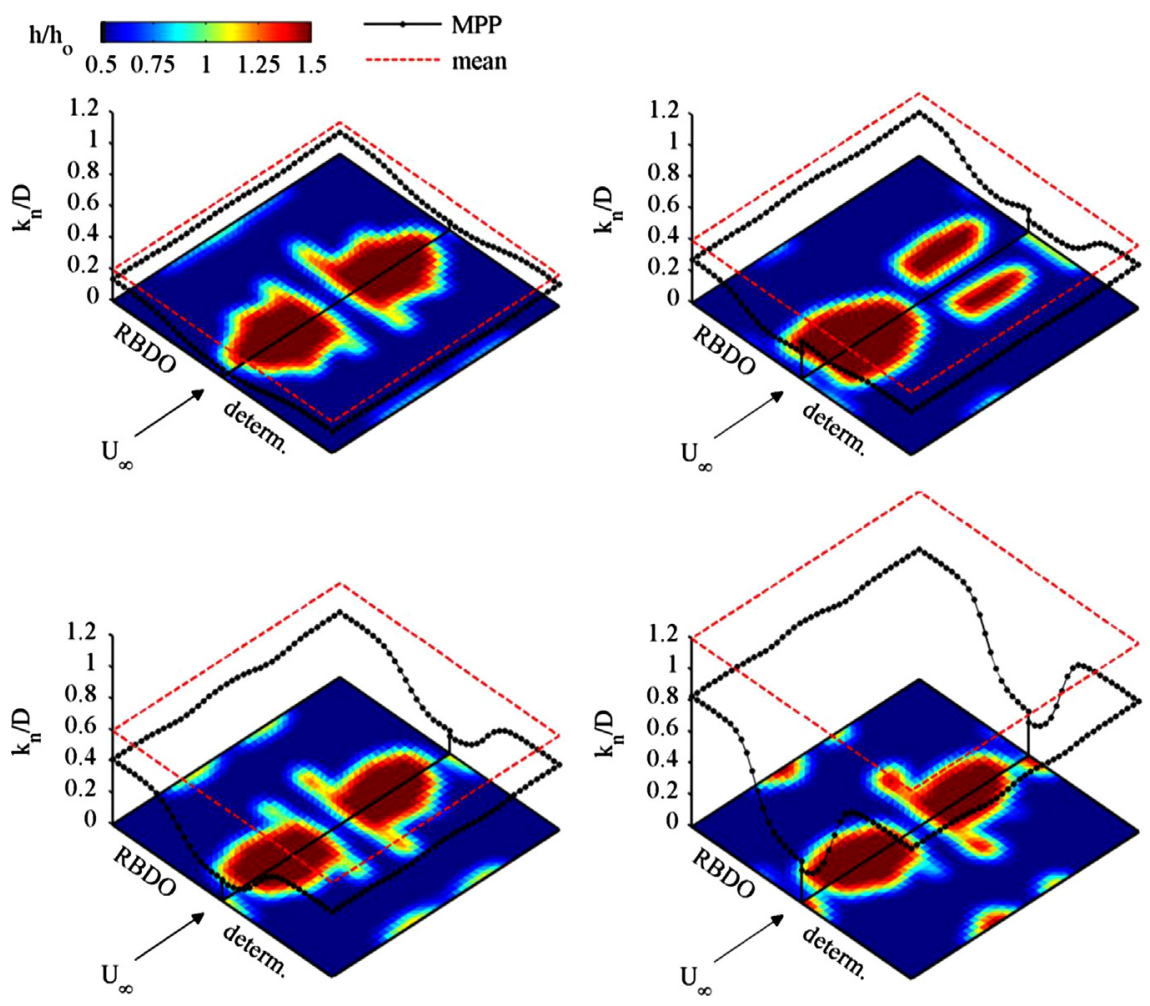

Fig. 9. Deterministic and reliability-based optimal thickness distributions, and their corresponding edge spring MPPs, for different scale factors $\alpha_{k} / D$.

these elements increases with the edge spring stiffness. The RBDO results remove this material almost entirely (a substantial topological change related to the advent of uncertainties), and reallocates it to increase the aforementioned high-thickness lobes. This is an interesting trend, given that elastic stresses will be very high at the panel edges (particularly for the nearly-clamped $\alpha_{k} / D=1.2$ case), but the optimizer has found a way to remove material here and also increase the deterministic flutter speed: inertial forces (M. $\ddot{\boldsymbol{u}}$ in Eq. (11)) would seem to play a large role, though additional factors are discussed below.

The MPP drawn around the perimeter of each panel is given in the physical space $\boldsymbol{r}=\left\{\begin{array}{llll}k_{1} & k_{2} & \cdots & k_{N}\end{array}\right\}^{T}$ rather than the standard normal space $(\boldsymbol{u})$. The vector norm of each MPP written in this space however, $\left\|\boldsymbol{u}_{\beta=\beta_{t}}^{*}\right\|$, is equal to 3 , which is the target reliability index for each RBDO case in Fig. 9. Only the corresponding probabilistic performance measures $g^{*}$ of the reliability-based optima are equal to zero; $g^{*}$ of each deterministic design is equal to some negative, infeasible (in the context of Eq. (28)) value. Each MPP is below the scale factor (or mean) $\alpha_{k} / D$, as decreasing the stiffness at the panel boundaries increases the likelihood of flutter, and the MPP for each case is qualitatively similar. The torsional stiffness along the panel sides ( $n_{\text {sides }}$ in Eq. (4)) is relatively high, with only minor undulations along this edge. This reinforces the conclusion made from Fig. 6 as well, that these nodes have a lesser role in determining the flutter speed of the panel.

Contrastingly, uncertainties in the boundary conditions along the leading and trailing edges $\left(n_{\mathrm{LE}}\right.$ and $n_{\mathrm{TE}}$ in Eq. (4)) are very important, with substantial drops in the MPP's torsional stiffness along these boundaries, particularly at the centerline. This potentially explains the reliability-based optimizer's willingness to remove material from this area (discussed above), as at the critical MPP, the boundary stiffness is low enough to emulate a simplysupported boundary condition, and so the edge stresses will be moderate. The MPP of the deterministic designs typically have slightly lower spring stiffnesses than their RBDO counterparts, though the leading edge of the $\alpha_{k} / D=0.4$ case is an exception. This is also the only case where the MPP along the leading edge and trailing edges are not largely the same, ostensibly because this thickness distribution has the greatest asymmetries between the leading half and the trailing half of the panel.

\section{RBDO: non-deterministic thickness design variables}

The final section of this paper considers the torsional boundary springs to be deterministic, prescribed, and spatially uniform around the perimeter of the panel, as in Section 3. Instead, the elemental thickness design variables themselves are assumed to be parameter-based aleatory uncertainties. Like the Weibull distributions for the edge springs, a non-normal distribution is needed for this case as well: negative panel thickness values are obviously infeasible, and substantial deviations from the intended distribution, though possible, are unlikely to pass visual inspections prior to incorporation into an aerospace vehicle. Again setting the design variables $\boldsymbol{x}$ as the thickness of each finite element, each member of the random variable vector $\boldsymbol{r}$ is uniformly distributed:

$r_{i}=U\left(x_{i} \cdot(1-\bar{h}), x_{i} \cdot(1+\bar{h})\right)$

where the mean value of each distribution is equal to the design variable $(\boldsymbol{x}=\boldsymbol{\mu}(\boldsymbol{r}))$, and the range of possible deviations (governed by $\bar{h}$ ) from this point is proportional to the mean. The value of $\bar{h}$ must be less than unity (to prevent negative thicknesses) and should be relatively small. The same value of $\bar{h}$ is used for each finite element, and so the coefficient of variation is constant across the panel.

As above, the random variables are statistically independent, but the thickness field is passed through a spatial filter prior to flutter computations, which provides some correlation. The FORM analysis is conducted in the standard normal space, and the 
transformation is

$\boldsymbol{u}=T(\boldsymbol{r}) \rightarrow u_{i}=\Phi^{-1}\left(\left(r_{i}-x_{i}+x_{i} \cdot \bar{h}\right) /\left(2 \cdot x_{i} \cdot \bar{h}\right)\right)$

Otherwise, the gradient-based optimization needed to find the MPP (Eqs. (26) and (27)) remain the same as in the previous section. The formulation of the RBDO problem (Eq. (28)) is the same as well, though the design gradients of the probabilistic performance measure, $d g^{*} / d \boldsymbol{x}$, must now reflect the fact that the design variables are non-deterministic:

$\frac{\mathrm{d} g^{*}}{\mathrm{~d} \boldsymbol{x}}=\left(\frac{\partial \mathrm{g}^{*}}{\partial \boldsymbol{r}} \cdot \frac{\partial \boldsymbol{r}}{\partial \boldsymbol{x}}\right)_{\boldsymbol{u}=\boldsymbol{u}_{\beta=\beta_{t}}^{*}}$

The two terms in this chain rule are both evaluated at the MPP $\boldsymbol{u}_{\beta=\beta_{t}}^{*}$. The first gradient is computed via the flutter derivative computation of Eq. (22), and the second is a diagonal matrix:

$\frac{\partial r_{i}}{\partial x_{i}}=(1-\bar{h})+2 \cdot \bar{h} \cdot \Phi\left(u_{i, \beta=\beta_{t}}^{*}\right)$

where $u_{i, \beta=\beta_{t}}^{*}$ is the $i$ th member of the MPP $\boldsymbol{u}_{\beta}^{*}=\beta_{t}$.

Two RBDO cases with non-deterministic design variables are given here. Both utilize a simply-supported boundary condition $\left(k_{n} / D=0\right)$, a target reliability index $\left(\beta_{t}\right)$ of 3.0 , and a thickness bound $\bar{h}$ of 0.2 (i.e., each thickness variable can vary by $\pm 20 \%$ from its mean value). One case utilizes the deterministic optimum for a simply supported plate (seen in Fig. 3) as an initial baseline design, as was done for each RBDO result in the previous section. The second case uses a uniform-thickness plate as a baseline, as was done for each case in Section 3. Both cases have the same critical flutter constraint, $\lambda_{c r}^{*}=533.9$. Similar exercises were conducted for stronger boundary conditions (higher values of $k_{n} / D$ ), but only the simply-supported case showed substantial topological differences between the deterministic and the reliability-based optimum, and any difference at all between the two different starting points.
Results for the first case can be seen in Fig. 10. The deterministic optimum baseline is repeated from Fig. 3, and the MPP (in the physical thickness space) of this design is given on the lower half of the panel. As would be expected, the location on the surface $\|\boldsymbol{u}\|=\beta_{t}$ which minimizes the flutter point largely involves a decrease in the thickness, as this weakens the panel. To form the MPP, up to $7 \%$ of the material is removed from the high-thickness lobes along the centerline, and a small amount $(\sim 1 \%)$ is taken from some variables already at the lower thickness bound $0.5 \cdot h_{o}$, though this is not visually evident from the figure. The reductions are less than the uniform bounds of each random variable (20\%), though the MPP is expected to approach these limits for larger values of $\beta_{t}$. Topological changes in the RBDO result for this case are largely confined to the trailing edge of the centerline, and the optimum's MPP has similar thickness reduction trends to the baseline's.

If a uniform thickness-plate is used as the initial baseline case however (Fig. 11), the reliability-based optimum is very different from the result of Fig. 10. The thin spanwise structures attached to the panel sides have been shortened considerably, and the leading and trailing halves of the panel are largely symmetric. A final dependence upon the starting point is a well-known characteristic of gradient-based optimization, but the simply-supported case is the only situation studied in which the two baseline designs did not converge to the same point. A comparison of the two convergence histories is given in Fig. 12. Data is displayed as above, where the flutter speed is plotted as a function of the panel volume, for both the deterministic mean structure and the MPP. An important difference between this plot and the data of Fig. 8 is that the MPP has a different volume (typically lower) than its corresponding mean design.

The convergence of the case with the deterministic optimum as a baseline is similar to cases given in the previous section: the

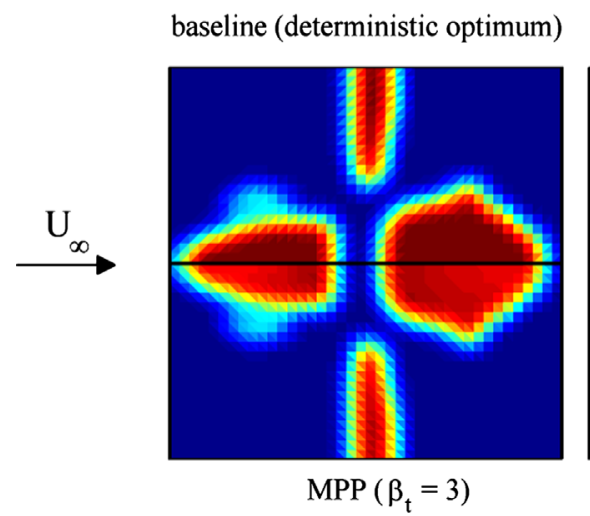

reliability-based optimum

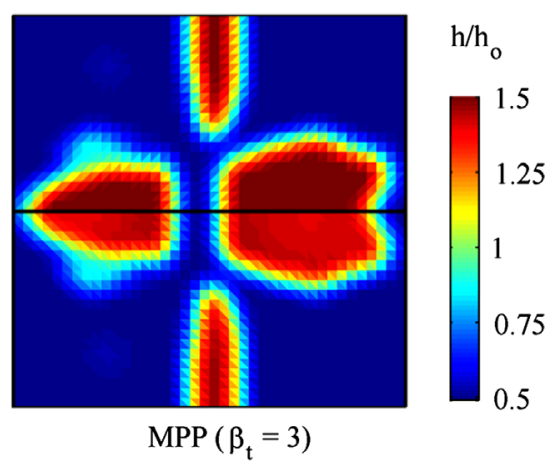

Fig. 10. Baseline panel thickness (deterministic optimum), reliability-based optimum, and their corresponding MPPs.

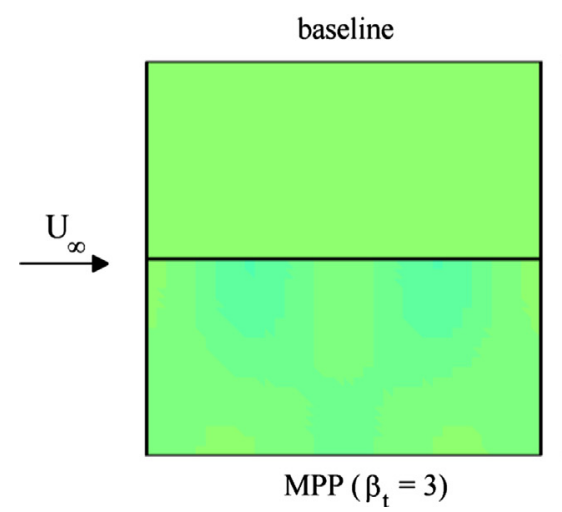

reliability-based optimum

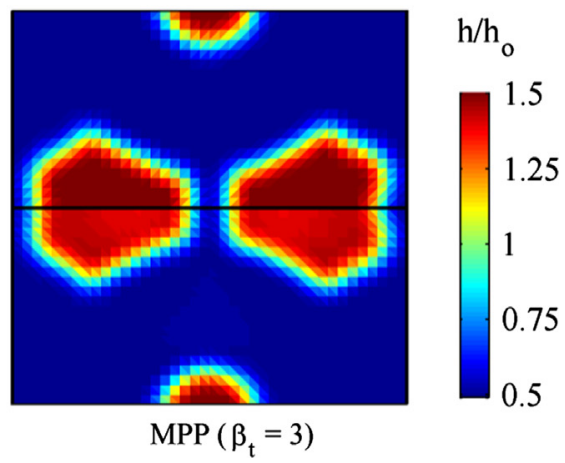

Fig. 11. Baseline uniform-thickness panel, reliability-based optimum, and their corresponding MPPs. 


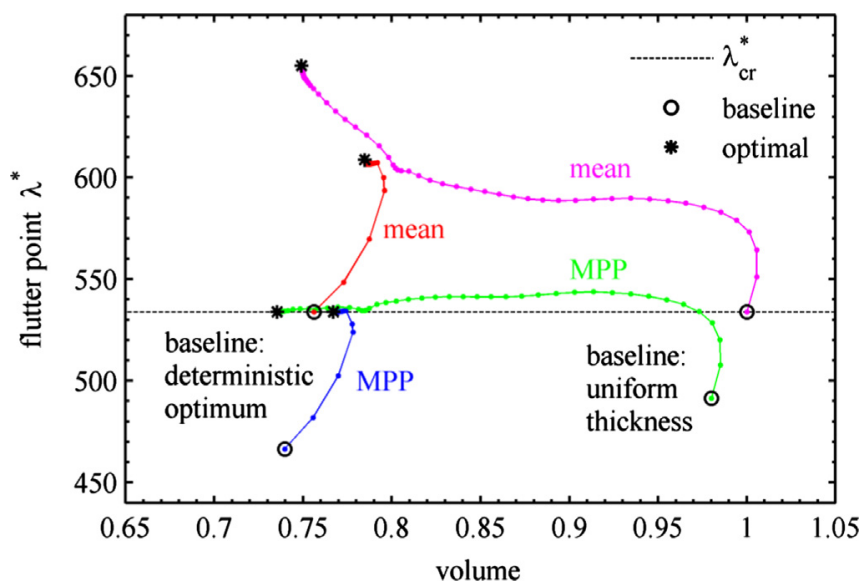

Fig. 12. Convergence histories for reliability-based design optimization with uncertain thickness distributions.

mean structure starts on the constraint boundary (533.9) and is pushed above it to 608.7, as the probabilistic performance measure $\mathrm{g}^{*}$ of the MPP is increased to zero. The inclusion of uncertainty in the thickness distribution has increased the mass of the panel by $3.87 \%$ over the deterministic design. The case with a uniformthickness baseline starts at a volume of unity (the MPP is $2.01 \%$ lighter), and its convergence slows as it reaches a very similar point in the design space as the previous case. The optimizer ultimately is able to move away from this area however, and converges to a structure with a volume that is $4.52 \%$ less. The deterministic flutter point increases substantially during the second half of this convergence process, and the final flutter margin is $22.88 \%$, the highest value of any of the cases in this paper. That this can occur for a structure with such a low mass is a testament to the highly complex nature of the non-deterministic thickness design space for panel flutter problems.

\section{Conclusions}

This paper has studied the structural optimization of a thin, square panel with high-speed supersonic flow over the top surface. The elastic surface is discretized into finite elements, and the thickness of each is utilized as a design variable. A deterministic gradient-based optimization problem is formulated that seeks to minimize the mass of the panel subject to an aeroelastic flutter speed constraint, and a series of frequency-separation constraints (which prevent the formation of higher-mode flutter points during the optimization process). A second optimization problem replaces the deterministic flutter constraint with a constraint on the maximum allowable probability that the panel will flutter (i.e., fail). For this RBDO exercise, the boundary conditions of the panel are parametrically uncertain. A series of torsional springs are placed along the panel's perimeter, each with a Weibull distribution governing its random stiffness. Failure probabilities are computed using FORM-based PMA methods. A final RBDO problem considers non-deterministic thickness design variables, each uniformly-distributed about a prescribed mean thickness. Results for each optimization study are given in terms of different mean values for the torsional springs along the perimeter (either prescribed or uncertain), and different critical flutter boundaries. In some cases, the introduction of uncertainties into the structural optimization problem provides a change in the topological distribution of stiffness and mass throughout the fluttering panel.

\section{Acknowledgments}

This work is sponsored by the Air Force Office of Scientific Research under Laboratory Task 03VA01COR, monitored by Dr. Fariba Fahroo.

\section{References}

[1] R. Bisplinghoff, H. Ashley, R. Halfman, Aeroelasticity, Addison-Wesley, Cambridge, MA, 1955

[2] C. Pettit, Uncertainty quantification in aeroelasticity: recent results and research challenges, J. Aircr. 41 (5) (2004) 1217-1229.

[3] B. Danowsky, J. Chrstos, D. Klyde, C. Farhat, M. Brenner, Evaluation of aeroelastic uncertainty analysis methods, J. Aircr. 47 (4) (2010) 1266-1273.

[4] N. Lindsley, C. Pettit, P. Beran, Nonlinear plate aeroelastic response with uncertain boundary conditions, Struct. Infrastruct. Eng. 2 (3) (2006) 201-220.

[5] L. Yi, Y. Zhichun, Uncertainty quantification in flutter analysis for an airfoil with preloaded free play, J. Aircr. 47 (4) (2010) 1454-1457.

[6] W. Stroud, T. Krishnamurthy, B. Mason, S. Smith, A. Naser, Probabilistic Design of a Plate-Like Wing to Meet Flutter and Strength Requirements, in: Proceedings of AIAA Structures, Structural Dynamics, and Materials Conference, Denver, CO, April 22-25, 2002.

[7] P. Attar, E. Dowell, Stochastic analysis of a nonlinear aeroelastic model using the response surface method, J. Aircr. 43 (4) (2006) 1044-1052.

[8] H. Khodaparast, J. Mottershead, K. Badcock, Propagation of structural uncertainty to linear aeroelastic stability, Comput. Struct. 88 (3) (2010) 223-236.

9] P. Beran, C. Pettit, D. Millman, Uncertainty quantification of limit cycle oscillations, J. Comput. Phys. 217 (1) (2006) 217-247.

[10] A. Manan, J. Cooper, Design of composite wings including uncertainties: a probabilistic approach, J. Aircr. 46 (2) (2009) 601-607.

[11] M. Ghommem, M. Hajj, A. Nayfeh, Uncertainty analysis near bifurcation of an aeroelastic system, J. Sound Vibr. 329 (16) (2010) 3335-3347.

[12] J. Meitour, D. Lucor, J. Chassaing, Prediction of stochastic limit cycle oscillations using an adaptive polynomial Chaos method, J. Aeroelast. Struct. Dyn. 2 (1) (2010) 3-22.

[13] N. Lindsley, P. Beran, C. Pettit, Integration of model reduction and probabilistic techniques with deterministic multi-physics models, in: Proceedings of AIAA Aerospace Sciences Meeting and Exhibit, Reno, NV, January 9-12, 2006.

[14] Z. Wang, Z. Zhang, D. Lee, P. Chen, D. Liu, Flutter analysis with structural uncertainty by using cfd based aerodynamic ROM, AIAA 2008-2197 (2008).

[15] R. Melchers, Structural Reliability Analysis and Prediction, Wiley, New York, 1999.

[16] D. Liaw, H. Yang, Reliability of initially compressed uncertain laminated plates in supersonic flow, AIAA J. 29 (6) (1991) 952-960.

[17] S. Castravete, R. Ibrahim, Effect of stiffness uncertainties on the flutter of a cantilever wing, AIAA J. 46 (4) (2008) 925-935.

[18] J. Yang, E. Nikolaidis, Design of aircraft wings subjected to gust loads: a safety index approach, AIAA J. 29 (5) (1991) 804-812.

[19] C. Verhoosel, T. Scholcz, S. Hulshoff, M. Gutiérrez, Uncertainty and reliability analysis of fluid-structure stability boundaries, AIAA J. 47 (1) (2009) 91-104.

[20] C. Pettit, R. Grandhi, Optimization of a wing structure for gust response and aileron effectiveness, J. Aircr. 40 (6) (2003) 1185-1191.

[21] M. Allen, K. Maute, Reliability-based design optimization of aeroelastic structures, Struct. Multidisciplinary Opt. 27 (4) (2004) 228-242

[22] M. Allen, K. Maute, Reliability-based shape optimization of structures undergoing fluid-structure interaction phenomena, Comput. Meth. Appl. Mechan. Eng. 194 (30) (2005) 3472-3495.

[23] M. Nikbay, N. Fakkusoglu, M. Kuru, Reliability-based aeroelastic optimization of a composite aircraft wing via fluid-structure interaction of high fidelity solvers, Mater. Sci. Eng. 10 (1) (2010) 1-10.

[24] B. Stanford, P. Beran, Computational strategies for reliability-based structural optimization of aeroelastic limit cycle oscillations, Struct. Multidisciplinary Opt. 45 (1) (2011) 83-99.

[25] J. Tu, K. Choi, Y. Park, A new study on reliability based design optimization, J. Mechan. Des. 121 (4) (1999) 557-564.

[26] B. Youn, K. Choi, An investigation of nonlinearity of reliability-based design optimization approaches, J. Mechan. Des. 126 (5) (2004) 403-411.

[27] E. Dowell, Nonlinear oscillations of a fluttering plate, AIAA J. 4 (7) (1966) $1267-1275$

[28] C. Mei, K. Abdel-Motagaly, R. Chen, Review of nonlinear panel flutter at supersonic and hypersonic speeds, Appl. Mechan. Rev. 52 (10) (1999) 321-332.

[29] T. Weisshaar, Aeroelastic optimization for a panel in high mach number supersonic flow, J. Aircr. 9 (9) (1972) 611-617.

[30] B. Peirson, Panel flutter optimization by gradient projection, Int. J. Numer. Methods Eng. 9 (2) (1975) 271-296.

[31] G. Van Keuren, F. Eastep, Use of Galerkin Method for Minimum-Weight Panels with Dynamic Constraints, Journal of Spacecraft 14 (7) (1977) 414-418.

[32] L. Beiner, L. Librescu, Minimum-weight design of an orthotropic shear panel with fixed flutter speed, AIAA J. 21 (7) (1983) 1015-1016.

[33] R. Barboni, A. Mannini, P. Gaudenzi, On the USe of the P-TFE method for panel flutter optimization, Comput. Struct. 70 (1) (1999) 109-117. 
[34] M. Langthjem, Y. Sugiyama, Optimum shape design against flutter of a cantilevered column with an end-mass of finite size subjected to a nonconservative load, J. Sound Vibr. 226 (1) (1999) 1-23.

[35] Y. Odaka, H. Furuya, Robust structural optimization of plate wing corresponding to bifurcation in higher mode flutter, Struct. Multidisciplinary Opt. 30 (2) (2005) 437-446.

[36] R. Haftka, Parametric constraints with application to optimization for flutter using a continuous flutter constraint, AIAA J. 13 (1975) 471-475.

[37] H. Ashley, G. Zartarian, Piston theory-a new aerodynamic tool for the aeroelastician, J. Aeronaut. Sci. 23 (12) (1956) 1109-1118.

[38] R. Cook, D. Malkus, M. Plesha, R. Witt, Concepts and Applications of Finite Element Analysis, Wiley, New York, 2002.

[39] A. Griewank, G. Reddien, The calculation of Hopf points by a direct method, IMA J. Numer. Anal. 3 (1) (1983) 295-303.

[40] S. Morton, P. Beran, Hopf-bifurcation analysis of airfoil flutter at transonic speeds, J. Aircr. 36 (2) (1999) 421-429.
[41] S. Timme, S. Marques, K. Badcock, Transonic aeroelastic stability analysis using a kriging-based schur complement formulation, AIAA J. 49 (6) (2011) 1202-1213.

[42] D. Murthy, R. Haftka, Derivatives of eigenvalues and eigenvectors of a general complex matrix, Int. J. Numer. Methods Eng. 26 (2) (1988) 293-311.

[43] K. Svanberg, The method of moving asymptotes-a new method for structural optimization, Int. J. Numer. Methods Eng. 24 (2) (1987) 359-373.

[44] T. Bruns, D. Tortorelli, Topology optimization of non-linear elastic structures and compliant mechanisms, Comput. Methods Appl. Mechan. Eng. 190 (26) (2001) 3443-3459.

[45] M. Rosenblatt, Remarks on a multivariate transformation, Annals of Mathematical Statistics 23 (1952) 470-472.

[46] M. Eldred, B. Bichon, "Second-Order Reliability Formulations in DAKOTA/UQ in: Proceedings of AIAA Structures, Structural Dynamics, and Materials Conference, Newport, RI, May 1-4, 2006.

[47] Y. Zhao, T. Ono, New approximations for SORM: Part 1, J. Eng. Mechan. 125 (1) (1999) 79-85. 